Cinémas

Revue d'études cinématographiques

Journal of Film Studies

\title{
La réception du film Independence Day en France. Un exemple de contre-acculturation?
}

\section{Laurence Alfonsi}

Volume 8, numéro 3, printemps 1998

Cinélekta 2

URI : https://id.erudit.org/iderudit/024756ar

DOI : https://doi.org/10.7202/024756ar

Aller au sommaire du numéro

Éditeur(s)

Cinémas

ISSN

1181-6945 (imprimé)

1705-6500 (numérique)

Découvrir la revue

Citer cet article

Alfonsi, L. (1998). La réception du film Independence Day en France. Un exemple de contre-acculturation? Cinémas, 8(3), 9-29.

https://doi.org/10.7202/024756ar
Résumé de l'article

Contrairement aux idées reçues, la mondialisation des échanges ne provoque pas une acculturation inéluctable des sociétés. C'est ainsi que la réception des films états-uniens en France est rarement identique au succès aux États-Unis. L'analyse porte ici sur la réception en France du film Independence Day (1996) qui connut un échec critique et des recettes très satisfaisantes, mais inférieures aux prévisions. Le film de Roland Emmerich ne suscita aucun débat esthétique et provoqua plutôt un rejet idéologique quasi unanime dans la presse française. En effet, celle-ci vit dans Independence Day le symbole même de l'impérialisme états-unien, faisant directement écho à un sentiment nationaliste traditionnellement vif en France. La réception d'Independence Day en France correspond donc à une réaction de contre-acculturation. 


\section{La réception du film Independence Day en France. Un exemple de contre-acculturation?}

\section{Laurence Alfonsi}

\section{RÉSUMÉ}

Contrairement aux idées reçues, la mondialisation des échanges ne provoque pas une acculturation inéluctable des sociétés. C'est ainsi que la réception des films étatsuniens en France est rarement identique au succès aux États-Unis. L'analyse porte ici sur la réception en France du film Independence Day (1996) qui connut un échec critique et des recettes très satisfaisantes, mais inférieures aux prévisions. Le film de Roland Emmerich ne suscita aucun débat esthétique et provoqua plutôt un rejet idéologique quasi unanime dans la presse française. En effer, celle-ci vit dans Independence Day le symbole même de l'impérialisme états-unien, faisant directement écho à un sentiment nationaliste traditionnellement vif en France. La réception d'Independence Day en France correspond donc à une réaction de contre-acculturation.

\section{ABSTRACT}

Contrary to the accepted view, the globalization of exchange does not produce an inevitable acculturation of societies. Thus the reception of American films in France rarely matches their success in the United States. This analysis looks at the reception of the film Independence Day (1996) in France, where it met with critical failure and where, though quite satisfactory, ticket sales were lower than forecast. In the French press, 
Roland Emmerich's film aroused no estheric debate, but provoked a nearly unanimous ideological rejection. Critics saw in Independence Day the very symbol of American imperialism, and their response echoed a nationalist feeling that has traditionally been very strong in France. The reception of Independence Day in France thus indicates a reaction of anti-acculturation.

Une œuvre d'art n'existe pas sans son créateur, mais n'existe pas non plus sans son public. Les études de réception s'appuient sur le présupposé d'une diversité des publics et donc de leurs attentes face aux événements représentés. Les spectateurs possèdent un "horizon d'attente", selon l'expression de Hans Robert Jauss, c'est-à-dire un système de valeurs qui résulte de l'expérience préalable - esthétique et sociale - et qui préside à l'interprétation de l'œuvre.

Cette étude se propose d'analyser la façon dont un film est reçu dans un contexte social, culturel et esthétique différent de son contexte d'origine. Pour cela, il est intéressant d'analyser l'accueil français ' d'un grand succès aux États-Unis. Mon choix s'est porté sur le film-événement de 1996, Independence Day (Roland Emmerich), dont la sortie commerciale s'effectua en France le 2 octobre 1996. Afin d'analyser la réception d'Independence Day en France, nous disposons de deux outils: les chiffres d'exploitation ${ }^{2}$ et le corpus critique, dont les articles proviennent de 18 journaux différents (revues de cinéma, hebdomadaires, quotidiens nationaux et régionaux).

À l'heure de la mondialisation des échanges, il devient presque banal de constater l'acculturation des sociétés. Alors qu'Independence Day connut un énorme succès aux États-Unis, comment la critique et les spectateurs français réagirent-ils face à ce film?

La réception du public états-unien et du public européen - UN SUCCES COLOSSAL AUX ÉTATS-UNIS

Independence Day pulvérisa le box office des États-Unis durant l'été 1996. Son démarrage fut fulgurant: 104,3 millions de dollars pour les six premiers jours, dépassant nettement les records précédemment établis par Mission impossible (74,9 millions de 


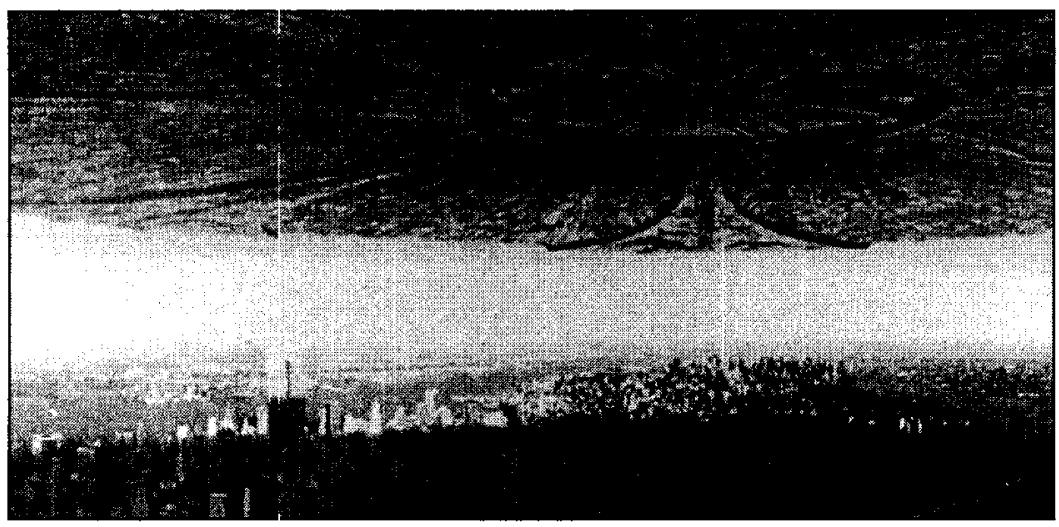

\section{Independence Day de Roland Emmerich (1996)}

dollars en six jours) et par Jurassic Park (74,1 millions de dollars). Independence Day, sorti aux États-Unis le 3 juillet (veille de la fête nationale, l'Independence Day), fut joué à guichets fermés. Il n'était pas rare de voir des spectateurs faire la queue pour la séance de quatre heures du matin devant des cinémas qui programmaient le film 24 heures sur 24.

Le succès d'Independence Day - le sixième film de l'histoire du cinéma états-unien en termes de recettes - fut aussi immédiat que durable ${ }^{3}$. Après 12 semaines, le film se situait toujours dans le Top Ten (neuvième position) et remonta même la semaine suivante à la sixième place. Pour sa quinzième semaine d'exploitation, les recettes hebdomadaires d'Independence Day étaient encore de 1296679 \$. Independence Day rapporta, en 26 semaines d'exploitation, $306137970 \$$.

\section{- LA RÉCEPTION DU PUBLIC EUROPÉEN : LES CHIFFRES D'UN SUCCÈS ANNONCÉ}

Au 29 octobre 1996, Independence Day avait généré 361 millions de dollars hors du territoire des États-Unis. En Europe, le succès d'Independence Day fut incontestable et se traduisit notamment par des débuts fulgurants ${ }^{4}$. En France, Independence Day, sorti dans 545 salles, attira pour la seule journée du 2 octobre 1996, 314000 spectateurs, dont 66415 sur Paris-périphérie. Comme prévu, le succès du film fut confirmé pendant la première semaine d'exploitation. Plus d'un spectateur sur deux vit

La réception du film Independence Day en France. Un exemple de contre-acculturation? 
Independence Day à Paris et dans sa périphérie. Après cinq semaines d'exploitation, Independence Day cumulait 5122402 spectateurs en France et dépassait le million sur Paris-périphérie.

\section{- UN SUCCESS EUROPÉEN SANS RÉSERVES ?}

Le grand succès d'Independence Day aux États-Unis devint un véritable phénomène de société. Le succès du film en Europe ne connut-il vraiment aucunes restrictions?

Pour comparaison, on peut citer l'exemple de Forrest Gump (Zemeckis, 1994) dont l'accueil français s'avéra nullement comparable au succès aux États-Unis ${ }^{5}$. Le 5 mars 1996, après 74 semaines, Forrest Gump avait attiré 895450 spectateurs à Paris. Ce chiffre reste inférieur au million d'entrées, ce qui pour un film états-unien à Paris est loin d'être un record. Par rapport à Forrest Gump, Independence Day a connu un succès nettement supérieur. Néanmoins, une comparaison est possible entre les deux films. Plusieurs films français diffusés en même temps que Forrest Gump connurent un nombre d'entrées supérieur ${ }^{6}$. Bien que ce ne soit pas le cas pour Independence Day, celui-ci n'éclipsa pas pour autant les films nationaux (ou européens) dans les différents pays de la communauté. C'est par exemple le cas en Italie, en Espagne et en Allemagne, où plusieurs films européens et nationaux connurent des résultats honorables ${ }^{7}$. En France, le succès d'Independence Day n'empêcha pas non plus les films français de résister à l' "offensive américaine». Lors de la première semaine d'exploitation d'Independence Day, neuf films français ou coproductions figuraient dans le classement des 15 meilleurs succès (le premier film de Pascal Bonitzer, Encore, est un exemple d'une belle résistance ${ }^{8}$ ). Bien que n'étant pas de gros succès, ces films permirent à la part de marché nationale de ne pas chuter face à l'arrivée d'Independence Day'.

L'évolution des réactions du public européen au cours des premières semaines d'exploitation d'Independence Day est encore plus significative. Rappelons que le succès du film aux ÉtatsUnis ne fut absolument pas un succès éphémère.

En Europe, bien qu'incontestable, le succès d'Independence Day ne fut pas aussi durable qu'aux États-Unis (par exemple, de façon plus ou moins marquée, en Italie, en Espagne, en Belgi- 
que ou en Allemagne) ${ }^{10}$. En France, Independence Day connut un excellent démarrage sans être tout à fait à la hauteur des prévisions". "Alors que la stratégie marketing faisait d'Independence Day un jackpot programmé aux États-Unis, les paris restent ouverts, du moins sur la longévité de sa carrière française", soulignait Le Film français ( ${ }^{\circ} 2631,20$ septembre 1996 , p. 3). En effet, le film présenta quelques difficultés à se maintenir sur la durée. En première semaine, sa part de marché était de $53 \%$, mais n'était plus que de $38 \%$ en deuxième semaine, de $26 \%$ en troisième et de $10 \%$ en quatrième. En deuxième semaine, les recettes d'Independence Day chutèrent de $40 \%$ sur Paris-périphérie et de $37 \%$ sur la France, une des plus grosses chutes des 15 meilleurs succès en France.

Le « raz de marée» fut donc inférieur aux prévisions (entre six et huit millions d'entrées selon les sondages). Independence Day, après 19 semaines, cumulait 5629113 millions d'entrées en France. Rappelons par exemple que Les Visiteurs (Poiré, 1993) cumula 13,6 millions d'entrées dans l'Hexagone ${ }^{12}$. C'est donc un fait : l'engouement du public français (voire même européen) pour Independence Day ne fut pas aussi exclusif et durable qu'aux États-Unis. On peut alors s'interroger sur l'origine des réactions du public français vis-à-vis d'Independence Day. Pour y répondre, nous mettrons en évidence les mécanismes de réception chez un spectateur particulier : le critique de cinéma.

\section{La réception critique d'Independence Day en France \\ - L'ABSENCE DE DÉBAT ESTHÉTIQUE}

Independence Day affiche une ambition évidente: s'appuyer sur des images inconscientes et sur des peurs primales universelles. Néanmoins, l'accueil du public français n'est pas aussi prévisible que la réception aux États-Unis, ni même comparable à cette dernière, au point que l'hebdomadaire Le Point écrit: "Tout le monde attend maintenant de voir quel sera en France l'effet Independence Day" ( $\mathrm{n}^{\circ} 1254,28$ septembre 1996, p. 132).

Le film, qui affiche une ambition consensuelle, entraîne aussi une réception unanime. Le rejet critique occulte le pluralisme habituel de la presse. "Un spectacle qui vise tous les publics, dans la grande tradition du genre", affirme Télé Star, journal 
populaire de télévision (30 septembre 1996, p. 120). «Les Américains ont vite reconnu dans Independence Day un vrai pop-corn movie, qu'on va voir en famille, sans réelle frayeur, sans sexualité ni violence graphique, avec des bons sentiments à la pelle, sans oublier que toutes les catégories de la population y sont représentées (et caricaturées de façon égale) ", écrit aussi le quotidien intellectuel Le Monde (3 octobre 1996, p. 27). "La niaiserie du scénario n'a d'égale que sa valeur symbolique et sociologique, dans sa culture du politiquement correct», réaffirme la revue cinématographique Positif (n 428, octobre 1996, p. 45).

En fait, le film ne parvient pas à susciter de débat esthétique. C'est cette absence qui explique largement l'échec critique d'Independence Day en France. Comme le souligne Le Monde, "Son apparente limpidité idéologique, sa linéarité narrative, la nullité de sa dramaturgie semblent décourager la réflexion en déployant sa puissance de machine industrielle" (3 octobre 1996, p. 27). Le film s'intègre parfaitement dans l'"horizon d'attente" d'un cinéma états-unien banal et conformiste. "ID4, rencontre du $3^{\text {ime }}$ archétype", titre La Marseillaise (2 octrobre 1996, p. 1). "Débauche d'images et de stéréotypes", titre également Le Monde (3 octobre 1996, p. 27). "Entre deux feux d'artifice, il y a vaguement une intrigue, mais si ténue, si javellisée [...]", souligne $L e$ Nouvel Observateur (n'1664, 26 septembre 1996, p. 114).

Pour une esthétique de la réception, la conscience réceptrice n'est pas contrainte à se réorienter vers l'horizon d'une expérience encore inconnue. En fait, aucun "changement d'horizon" n'est nécessaire pour assimiler l'œuvre. Independence Day confirme les expériences déjà acquises du public. "Le film ne fonctionne que sur des effets de reconnaissance. [...] Les clichés défilent et rassurent ", souligne Le Monde (3 octobre 1996, p. 27).

Plus précisément, Independence Day procède à un amalgame de genres hollywoodiens célèbres. Il pastiche notamment les séries $B$ de science-fiction, très populaires dans les années cinquante, et les films catastrophes des années soixante-dix, plaçant ainsi le public sur un terrain familier. La Marseillaise compare Independence Day à un " [...] phénomène sampler bien connu des inconditionnels de Dance music: les genres cinémato- 
graphiques (la science-fiction, le film de guerre, le film catastrophe) sont mixés, étirés, accumulés en strates répétitives légèrement (et graduellement) décalées donnant à l'ensemble une sorte de cohérence hypnotique" (2 octobre 1996, p. 20). "Ce n'est plus du cinéma, c'est du Lego informatique», note également François Forestier (Le Nouvel Observateur, n ${ }^{\circ} 664$, 26 septembre 1996, p. 115).

Il est certes vrai que les réticences des critiques français prennent pour base une certaine mythologie romantique de l'artiste, la tradition française de l'art pour l'art. La conséquence est l'association presque naturelle entre succès commercial et médiocrité dans un pays franciscain où l'argent éveille forcément la méfiance. Logiquement, une telle approche cinématographique se retrouve dans les commentaires virulents des Cahiers $d u$ cinéma. La revue insiste sur l'incohérence de la carrière de Roland Emmerich. Bien qu'ex-étudiant de l'École de cinéma de Munich, le cinéaste est perçu comme subissant "l'influence inhibante de Steven Spielberg et George Lucas" :

Entre enjeux commerciaux et idéal du moi cinéphilique, le point sur le nouveau film d'action hollywoodien. [...] Mais qui est au juste Roland Emmerich? Les journalistes américains, après lavage de cerveau par la campagne marketing de la Fox, n'ont pu rendre compte d'autre chose que du succès imminent d'Independence Day. Parmi les péchés par omission, personne n’a fair la démarche élémentaire de jeter un oil au travail antérieur de Roland Emmerich. [...] Il a critiqué l'ingérence américaine dans la politique du Moyen-Orient dans son premier film, Das Arche Noab Prinzip (1984) - une prise de position qui était peut-être moins liée à des convictions profondes quau fait que ce film étair en partie financé par le Fonds pour le jeune cinéma allemand. Du moins est-ce la seule explication que je puisse trouver au fait que, 12 ans plus tard, il ait réalisé un gros succès fondé sur le programme politique républicain de 1992 et produit par l'ultra-conservateur Rupert Murdoch. [...] Pour être juste envers Emmerich, il n'a probablement pas eu besoin de retourner sa veste: la surenchère entre les quatre studios hollywoodiens qui voulaient produire Independence Day était 
devenue inévitable après le succès surprise de Stargate pour MGM-UA (Cabiers du cinéma, n" 505, septembre 1996, p. 55 et 61).

Une longue démonstration qui se résume à la classique opposition entre logique du producteur et logique de l'auteur. Pourtant, le rejet de la critique face à Independence Day dépasse cette tradition française de l'" auteurisme». Confrontons pour cela les réflexions des Cahiers du cinéma et de Positif, qui possèdent deux politiques éditoriales différentes, traditionnellement opposées. Les Cahiers du cinéma insistent sur la mise en scène, la théorie de l'auteur, tandis que Positif privilégie les sujets et reste plus favorable au cinéma hollywoodien. Pour Positif, Independence Day déçoit, non pas par rapport à ce qu'il n'est pas (un film d'auteur), mais par rapport à ce qu'il devrait être (un film américain à grand spectacle) : "Côté effets spéciaux, tout est "grillé" durant la prometteuse première heure, de loin la plus excitante, avec de jolies explosions de l'Empire State et de la MaisonBlanche. La répétitivité s"installe dans les dernières bobines" (n" 428, octobre 1996, p. 45). La justification du rejet differe de celle des Cahiers du cinéma, mais mène au même résultat: le rejet du film sans débat esthétique.

Ceux qui voient dans Independence Day un pur spectacle de science-fiction reprochent alors au film sa démesure. Fait d'autant plus significatif, ces critiques émanent même de magazines "populaires" de télévision, habituellement plus cléments envers les hits états-uniens: "Une nouvelle aventure de science-fiction dont la démesure naïve, spectaculaire et patriotique mérite les superlatifs", note Télé Star (30 septembre 1996, p. 120). "Du cinéma qui veut nous en donner pour nos sous jusqu'à l'indigestion", souligne également Télé Loisirs ( $\mathrm{n}^{\circ} 533,30$ septembre 1996). Finalement, le rejet d'Independence Day s'effectue quel que soit le mécanisme de réception (jugement par rapport à ce que le film est, n'est pas ou devrait être).

Le magazine Variety présenta Independence Day comme la plus grande des séries B de toute l'histoire du cinéma. À l'opposé, les Cahiers du cinéma affirment:

L'une des choses les plus irritantes dans les critiques américaines de ce film, c'est que leurs auteurs, pour la 
plupart, excusent les absurdités du scénario comme étant dans la tradition de ses antécédents, les séries $B$ des années 50 , qui dans leurs souvenirs sont nombreux et uniformément patriotards. [...] La majorité des critiques de cinéma américains sont dénués d'un quelconque sens de l'histoire (n" 505, septembre 1996, p. 60).

Contrairement aux affirmations des Cahiers du cinéma, aucune hiérarchie, sur un plan esthétique, ne peut être faite entre la critique française et la critique états-unienne. Chacune trie les informations qu'elle reçoit en fonction de son «horizon d'attente", marqué par les diverses influences subies. La réception est en cela une théorie de l'interaction, qui distingue « [...] ce qui relève de la situation et ce qui est en situation" (Goffman, p. 208) ${ }^{13}$. En l'occurrence, la réception critique d'Independence Day en France est marquée par l'omniprésence des jugements idéologiques, ou plutôt du jugement idéologique, tant celui-ci est également unanime.

Évidemment, on pourrait souligner que l'absence de débat esthétique en France s'explique logiquement par le manque de qualité artistique du film. Néanmoins, bien que cette remarque soit en partic justifiće, l'appréciation de la qualité d'un film résulte toujours de critères relatifs qu'il est souvent inintéressant, voire dangereux, de hiérarchiser. D'autre part et surtout, l'absence de débat esthétique suscité par Independance Day en France participe en fait d'une réflexion sociologique majeure sur l'avenir du pluralisme social et culturel. Cette réflexion représente l'intérêt central de la présente analyse et justifie le choix d'Independance Day pour une étude de réception dans l'Hexagone.

\section{- LES EFFETS APPARENTS}

\section{D'UNE CONTRE-ACCULTURATION}

Le succès d'Independence Day aux États-Unis ne s'explique pas uniquement par la puissante campagne de marketing mise en œuvre. D'autres films firent l'objet d'une publicité plus importante sans obtenir de telles recettes (Mission impossible). Independence Day a su combler une attente toujours potentielle chez son public immédiat, celle de l'impérialisme. Les extraterrestres, qui veulent envahir la Terre en exterminant ses habitants, 
deviennent des symboles de tous les ennemis passés ou potentiels des États-Unis. "Comme ces extraterrestres-là sont résolument destructeurs, les anéantir est une mission politiquement correcte, certaine de réjouir le public à l'unanimité. Le paranormal a devancé le communisme sur l'échelle de la paranoïa américaine» (Le Monde, 3 octobre 1996, p. 27).

Le concept même d'Independence Day - une invasion d'extraterrestres pour la fête de l'indépendance des États-Unis demeure forcément étranger au public français. Contrairement aux spectateurs états-uniens, la publicité française ne put jouer sur l'idée - toujours indispensable - d'une fiction ancrée dans la réalité. Aussi utilisa-t-elle un slogan fondé sur la phobie universelle des extraterrestres et sur l'attrait d'une riposte spectaculaire.

Pourtant, la reconnaissance de concepts universels ne constitue pas un processus de réception très présent dans la critique française. L'identification de la provenance du film est constante. De la sorte, l'effet cathartique du film, et par extension son pouvoir attractif, semble être limité au seul peuple des ÉtatsUnis. C'est ce que constate l'ensemble de la critique française, qui ironise largement sur l'ampleur du succès d'Independence Day aux États-Unis. L'effet cathartique du film concerne d'abord le passé historique de l'Amérique, notamment les événements douloureux des années soixante, qui mirent à mal le mythe de l'American way of life.

Face à l'énorme succès d'Independence Day aux États-Unis, la critique française, dans son ensemble, s'est montrée particulièrement sévère quant au message véhiculé par le film. "Ce serait une erreur de mésestimer ce qu'ID4 induit et comporte, idéologiquement, sous couvert de spectacle spectaculaire" (La Marseillaise, 2 octobre 1996, p. 20). "Morale douteuse garantie", souligne François Forestier dans Le Nouvel Observateur (n"1664, 26 septembre 1996, p. 114).

La critique française rejette avant tout la philosophie «globalisante" véhiculée par le film, c'est-à-dire une conception qui annihile et uniformise les différences à la fois culturelles et individuelles. Conforté par des stéréotypes révélateurs, le mythe de la nation états-unienne repose sur le melting pot d'individus 
valeureux, en l'occurrence un Juif et un Noir. «Le premier, forcément, est intelligent et trouve le virus informatique qui va trouer la grosse galette. Le second, forcément, est brave (il capture un extraterrestre et sauve au passage la First Lady) et facétieux" (Télérama, $\mathrm{n}^{\circ}$ 2438, 2 octobre 1996, p. 32). Autre stéréotype décelé par la critique: la méfiance toujours justifiée envers l'Europe de l'Est, souvenir de la "paranoïa " communiste des États-Uniens :

Signalons au passage que cetre belle victoire de l'Amérique - pardon, du monde - a été remportée malgré un affreux personnage, le secrétaire à la Défense, Nimziki. Comme quoi, 40 ans après la guerre froide et sept ans après la chute du Mur, on ne se méfie jamais assez des Slaves, même lorsqu'ils ont la nationalité américaine (Télérama, n" 2438, 2 octobre 1996, p. 32).

La représentativité du politically correct se traduit alors par des victimes marginales. «Parmi les premiers à périr sous le feu des E.T., on note un homosexuel, un alcoolique, une féministe et un sniffeur!» (La Marseillaise, 2 octobre 1996, p. 20). "Le gay et le savant fou seront sacrifiés à l'autel de la singularité ", ajoute Positif (n" 428, octobre 1996, p. 45). C'est donc bien une philosophie du rejet de l'étranger que dénigre la critique française. «Il est parfaitement logique que le président-sauveteur se nomme Whitmore puisque son but est de lessiver toujours plus blanc les basanés de l'espace", ironise Libération (2 octobre 1996, p. 29).

Le rejet d'une telle philosophie prend ses sources dans une culture et un contexte bien particuliers: ceux de l'affaire des Africains "sans papiers " ${ }^{14}$ sur fond de débat sur l'extrême-droite et sur le racisme. C'est ainsi que la critique française "resitue" le discours sur le film dans une perspective française:

Pour mesurer la puissance comique enfouie dans Independence Day, il suffit d'imaginer une transposition hexagonale de l'affaire. Disons un prochain film de JeanJacques Annaud, intitulé 14 juillet, avec Jacques Chirac boutant hors de France les Martiens sans papiers aux commandes de son magical Mirage tandis que Bernadette agoniserait dans les ruines fumantes de l'Élysée.

Poilade assurée" (Libération, 2 octobre 1996, p. 29). 
La culture réceptrice réinterprète le film en fonction de ses propres valeurs, dans un contexte donné, et lui attribue des significations spécifiques par rapport au contexte d'origine. C'est toujours à partir de la double perception de similitude et d'altérité que se mettent en place les mécanismes de réception. Les différentes tentatives d'assimiler une altérité conformément aux normes de l'imaginaire collectif, en l'occurrence français, témoignent de la nécessité de relativiser la thèse de la "déculturation" (selon l'expression de Pierre Bourdieu) censée découler de la mondialisation. La présence des références nationales est le signe que, même dans le cas de la culture états-unienne prétendue universelle, une cuvre n'est jamais directement assimilable et convaincante.

C'est d'autant plus vrai que le rejet de la philosophie "globalisante" proposée par le film correspond en fait à un problème qui dépasse celui du racisme en France. Plus largement, le refus de l' "autre", tel que la critique française envisage Independence Day, s'inscrit dans le débat sur la mondialisation et sur les dangers du modèle unique véhiculé par les États-Unis dans le monde. La réflexion suivante du Nouvel Observateur témoigne bien de cette étroite relation effectuée entre un racisme national et international :

Lidéologie d'Independence Day est assez nauséabonde. Sans l'informatique et l'Amérique, nous serions des esclaves. Des traîne-savates dans un zoo cosmique. Des sans-papiers, vous vous rendez compte. C'est l'hymne grandiose à la technologie américaine, le chant de la liberté de Silicon Valley. Le principe de base est un tantinet inquiétant : tout ce qui vient d'ailleurs est méchant. Méfiez-vous des immigrés (n"1664, 26 septembre 1996, p. 114).

Le refus de la "globalisation" est bien au cour des commentaires critiques. Preuve en est, le rejet du film - et plus largement de la philosophie "mondialiste" qu'il véhicule — s'effectue quelles que soient les appartenances idéologiques des journaux. Le film est systématiquement rendu non compatible avec la tendance politique du journal, de sorte que l'interprétation se réduit à un processus de contre-assimilation. Prenons 
pour exemple L'Express et les Cahiers du cinéma. Tous deux different à la fois par leur coloration idéologique et par leur nature (hebdomadaire d'information générale et revue de cinéma). L'Express perçoit Independence Day comme un film démocrate, ce qui idéologiquement ne correspond pas véritablement à l'hebdomadaire et appuie ainsi la dépréciation: "Independence Day, film coqueluche de l'Amérique, fait campagne pour Bill Clinton. [...] Independence Day dessine l'arche de Noé du millénaire: Blancs, Noirs, Mexicains, Juifs, gays, etc., unis vers Clinton. Une affiche électorale cousue main" $\left(\mathrm{n}^{\circ} 2360,26\right.$ septembre 1996, p. 112-113).

Par contre, les Cahiers du cinéma voient dans Independence Day un film de droite et républicain, à l'instar des propos tenus par le réalisateur ${ }^{15}$ :

L'idéologie d'Independence Day est manifestement de droite. Le président est un sosie de Bush et a de toute évidence les mêmes idées. [...] Le seul point commun avec Clinton est la jeunesse. [...] Bob Dole, candidat républicain à l'élection présidentielle, a laconiquement vanté le film en quittant la salle ( Un bon film à voir avec votre famille et dont on peut être fier. Il parle de la diversité en Amérique. Du leadership»), et l'a ensuite cité en exemple, avec Forrest Gump et Braveheart, comme le type de films que Hollywood devrait produire davantage (Cabiers du cinéma, n" 505, septembre 1996, p. 60-61).

La réception critique s'oppose alors à la tradition gauchiste des Cahiers du cinéma. À partir des années soixante, la revue découvre en effet les nouveaux cinémas nationaux et se lance dans les luttes politiques (l'affaire Langlois, Mai 68, l'engagement gauchiste, soutien des mouvements sociaux aux États-Unis comme la contre-culture californienne, etc.). Lorientation de la revue, devenue un symbole des luttes anticonformistes et des cinémas indéperidants, conduit naturellement à marquer l'incompatibilité entre la vision d'Independence Day et la "culture " réceptrice.

Dans Independence Day, l'Europe et le tiers-monde sont en effet relégués au rang d' "objectifs collatéraux». Ce n'est que 
tardivement et brièvement que l'on apprend que les extraterrestres ont également touché Londres, Paris, Rome et Plan-deCuques. L'écran est presque entièrement occupé par les destructions à Washington, à New York et à Los Angeles. "L'Amérique est le monde" affirment les Cahiers du cinéma (n" 505, septembre 1996, p. 60). L'histoire d'Independence Day - l'invasion d'extraterrestres - devient le symbole indiscutable de l'«américanisation $»$ du monde :

Un film porteur d'une propagande américaine ( $L e$ Canard enchaîné, n" 3962, 2 octobre 1996, p. 6).

Hollywood impose le nouvel ordre planétaire (Télé$m a m a$, n" 2438, 2 octobre 1996, p. 32).

Laveu naîf d'un tel fantasme (toute la planète à l'heure des États-Unis) est sans doute l'expression la plus voyante d'un message guère subtil (Le Monde, 3 octobre 1996, p. 27).

"Il " débarque. Avec 550 copies, ses effets spéciaux et ses propos spécieux, Independence Day envahit nos écrans. [...] Lorsqu'ils débarquent sur notre bonne vieille terre, c'est pour nous envahir, nous coloniser, nous bouffer, nous réduire en esclavage ( $\mathrm{La}$ Marseillaise, 2 octobre 1996, p. 1 et 20).

Tous les instruments de la mondialisation présents dans le film alimentent ainsi l'ironie ou la virulence des critiques. Les outils de communication en font principalement partie dans la mesure où l'arme du film est l'intelligence d'un citoyen muni d'un ordinateur. "ID4 confirme que le prochain millénaire appartiendra aux citoyens de l'Internet!» (Le Monde, 3 octobre 1996, p. 27). "Independence Net: http://www.id4.com" (L'Express, $\mathrm{n}^{\circ}$ 2360, 26 septembre 1996, p. 112). "Pour la suite, voir http://www.id4.com/ et tourner à gauche" (Le Nouvel Observateur, n"1664, 26 septembre 1996, p. 114).

La critique utilise souvent des expressions empruntées au vocabulaire guerrier ou judiciaire. Libération souligne que les dénonciations constituent des "réflexes de légitime défense" qui conduisent même à sympathiser avec les extraterrestres, contre les États-Uniens : 
Le même réflexe devrait inciter à prendre en symparhie immédiate les Martiens que le film prend littéralement en grippe. [...] La pyrotechnie du début est un instant régalante puisqu'elle figure, entre autres, l'explosion de la Maison-Blanche (mais pas celle, hélas, d'Eurodisney) (2 octobre 1996, p. 29).

Finalement, les critiques françaises sur Independence Day pourraient se résumer par le slogan lancé par le quotidien $L a$ Marseillaise: "À chacun, lucide, d'entrer en Résistance» (2 octobre 1996, p. 20).

Independence Day est donc assimilé à un phénomène de "déculturation" dont serait victime un pays occupé en temps de guerre. Les Cahiers du cinéma soulignent que «[...] le film appelle à l'union mondiale. [...] Bush a appelé cela "le nouvel ordre mondial", mais même lui n'a pas eu le culot de suggérer que le reste du monde, ayant accepté la domination de l'Amérique, devrait adopter sa fête nationale" ( $\mathrm{n}^{\circ} 505$, septembre 1996, p. 61) ${ }^{16}$. Télérama reprend cette image sur le mode de l'ironie:

14 juillet. Bye-Bye Hollywood, revenons sur terre. Une irréductible tribu s'obstine à célébrer sa fête locale. On apprend que d'autres peuplades, qui n'ont pas bien compris le message d'Independence Day, feraient de même à d'autres dates de l'année. C'est toujours pareil : les Américains font le sale boulot, il y en a toujours qui manquent de reconnaissance.

2 octobre. Épilogue. Hollywood, qui a planifié l'invasion, envoie Independence Day chez les irréductibles (n" 2438, 2 octobre 1996, p. 32).

\section{- AU-DELÀ D'UNE CONTRE-ACCULTURATION}

Le cinéma représente un outil de recherche intarissable pour comprendre les faces cachées des sociétés, germes des évolutions sociales. Ainsi, le terme de "résistance", tel qu'il a pu être utilisé ou connoté dans la réception française d'Independance Day, est au cour des mutations sociales et culturelles. C'est pourquoi, il est plus que jamais pertinent de poser la question des formes actuelles de résistance sociale et artistique.

Dans un univers de plus en plus fortement médiatisé et donc dirigiste, le spectateur peut-il encore exercer sa liberté d'inter- 
prétation? Cette question est particulièrement présente dans le marché cinématographique, où la culture du spectacle, véhiculée par les productions hollywoodiennes, tente de fournir une mémoire collective à l'humanité. Pourtant, parallèlement, le pluralisme postmoderne nous amène aussi à réfléchir sur la liberté d'un spectateur de plus en plus avide de champs artistiques nouveaux et variés.

Si les mutations actuelles suscitent des sentiments contradictoires, voire ambigus, c'est notamment que la mondialisation a pour effet paradoxal de réduire les macrosystèmes classiques qui obéissaient aux règles du progrès moderne fondé sur l'unification rationnelle du monde. Les sociétés de la postmodernité se caractérisent en effet par le redéploiement de diverses formes de sociabilité. L'individu devient de plus en plus "pluriel ", produit et producteur de relations sociales diverses. La culture s'impose comme un catalogue "[...] dont les acteurs se servent pour renégocier en permanence leur identité" (Amselle, p. 10). "Le soi n'est donc pas une entité à moitié dissimulée derrière les événements, mais une formule changeante pour se gérer à travers eux" (Boltanski, p. 208). Nos sociétés postmodernes développent ainsi une flambée différentialiste, un accroissement fulgurant des valeurs et des modes de vie. Ce que le sociologue français Michel Maffesoli appelle "l'ère des tribus" correspond à des sociétés polymorphes qui sont prises dans un mouvement effervescent de constante transformation.

Face à cette confrontation permanente des valeurs, l'individu n'est pas forcément voué à perdre son libre arbitre. Soulagé du poids des inflexibles caractérisations sociales et devenu de plus en plus sélectif, il pourrait acquérir davantage de liberté. Ce surcroît de liberté et d'imagination entraîne également un surcroît d'incertitudes. Or, ce sont bien des conflits et des doutes que naissent les prises de conscience, puis les actions individuelles et collectives. Toute construction — d'une identité ou d'une légitimité - est autant une construction "pour" qu'une construction "contre" dans la mesure où l'homme ne bâtit et n'évolue qu'en doutant, qu'en confrontant. D'où les limites avérées des approches rationnelles, qui supposent un univers prédictible et contrôlable. 
La réception d'Independence Day auprès de la critique française "contredit " et "veut contredire" ce rapport entre mondialisation et uniformisation rationnelle des attentes. Independence Day devient alors le symbole inacceptable de la pensée unique et de l'impérialisme états-unien. Néanmoins, si l'on inscrit la réception française d'Independance Day dans les mutations de nos sociétés postmodernes, de telles réactions "différentialistes" ne se réduisent plus à de simples mouvements épars de contre-acculturation.

Cette analyse, centrée sur un exemple particulier, est nécessairement incomplète. Elle appartient à une problématique qui demande à être étayée à l'aide d'un corpus plus important de films et surtout à l'aide de méthodologies nouvelles. Les mutations actuelles encouragent en effet un nouveau mode de savoir, transdisciplinaire. La transdisciplinarité connaît un essor logique et correspond à un mode de pensée complexe adapté aux enjeux du XXI siècle.

Cet essor de la transdisciplinarité s'accompagne du retour en force du «sujet", qui avait été marginalisé par la science disciplinaire. Un des sujets transdisciplinaires, dont l'étude nous plonge au cour des mutations socio-culturelles, est justement le processus de résistance, qui connaît lui aussi des changements. C'est par exemple le cas du développement des "microrésistances" qui sont des résistances minoritaires (ce qui ne signifie pas inefficaces), spontanées et/ou éphémères.

Autant de mutations et d'enjeux qui nécessitent l'élaboration de véritables méthodologies et applications de recherche autour du concept de « résistance».

Université d'Aix-Marseille I

\section{NOTES}

1 Nous utiliserons également quelques exemples relatifs à la réception d'Independence Day dans les autres pays européens.

2 Communiqués par Le Film français et par différents organismes européens de statistiques.

3 Évolution des recettes au cours des différents mois d'exploitation d'Independence Day.

4 En Belgique, Independence Day réussit la meilleure première semaine d'exploitation depuis 1986 (dépassant donc le succès de Jurassik Park) et s'appropria 50\% des 
parts de marché. Le film récolta $33430 \$$ après une semaine à Bruxelles. En Espagne, Independence Day cumula, après 10 jours d'exploitation, 1963307 spectateurs. En Italic, Independence Day attira, après la première semaine d'exploitation, $4536468 \$$, et après la deuxième semaine, $6304524 \$$, ce qui plaçait le film nettement au-dessus des autres films exploités (Mission impossible, $888391 \$$ après quatre semaines). En Allemagne, Independence Day connut également un début fulgurant (quatre millions d'entrées après deux semaines d'exploitation).

5 Pour plus de détails, on se référera à mon article intitulé "La réception du film Forrest Gump (Robert Zemeckis) en France. Vers une remise en cause du mondialisme culturel », publié dans les Cabiers de sociologie économique et culturelle en juin 1996.

6 Par exemple, Un Indien dans la ville (1 175957, 64 semaines), Le bonheur est dans le pré (1 006095 après seulement 13 semaines), Les Trois Frères (941 084, seulement 12 semaines).

7 En Italic, parallèlement à la première semaine d'exploitation d'Independence Day, Bambola, une coproduction européenne de Bigas Luna, se plaça en excellente troisième position. Deux films italiens se situent également parmi les 10 premiers, La Lupa $\left(6^{\circ}\right)$ et Vesna va veloce $\left(9^{\circ}\right)$. Lors de la seconde semaine d'exploitation d'Independence Day, une comédie italienne signée Christian de Sica, Tre-3, connut un bon démarrage (7 position avec $416258 \$$ ). Une semaine plus tard (semaine du 7 au 13 octobre 1996), Le Huitième Jour (film français) entra dans le Top Ten ( $8^{\circ}$ place). Durant la semaine du 14 au 20 octobre 1996, les films états-uniens affirment leur hégémonie avec huit films dans le Top Ten. Néanmoins, la semaine suivante, comme une réponse à l' "invasion américaine", Il Barbiere di Rio se place en seconde position.

En Espagne, alors qu'Independence Day bat tous les records de fréquentation sur son premier week-end, deux films espagnols se classent dans le Top Ten, Los Porretas et Tengo una casa. De même, dans la semaine du 21 au 27 octobre 1996, malgré une mauvaise critique, Bambola se classe en huitième position.

En Allemagne, après sept semaines, Independence Day était second au classement. Cola n'empêcha pas la belle performance du film allemand Irren ist männlich ni l'entrée remarquée du film français Microcosmos à la neuvième place avec seulement 53 copies.

8 Encore est le premier film de Pascal Bonitzer, sorti une semaine avant Independence Day. Il réalisa lors de sa première semaine 28471 entrées sur 13 salles et la deuxième meilleure moyenne par écran de la semaine (2190 spectateurs). En deuxième semaine, Encore ne perdit que $7 \%$ par rapport à sa première semaine d'exploitation en France.

9 Le cinéma français résista moins bien face au film Mission impossible. Sa part de marché baissa à $17 \%$ sur Paris-périphérie, alors qu’il résistait mieux face à Independence Day (20\% de part de marché la semaine de sa sortie). Il faut dire que Mission impossible avait quelques atouts supplémentaires par rapport à Independence Day (une distribution avec plusieurs comédiens français, un réalisateur célèbre -- Brian De Palma - notamment auprès des cinéphiles).

10 En Italie, Independence Day resta trois semaines en tête du box office, avec une chute très nette lors de la troisième semaine $(6304524 \$$ en deuxième semaine et $1593815 \$$ en troisième semaine). Le film disparut assez vite du Top Ten ( $5^{\circ}$ en quatrième semaine et $7{ }^{\circ}$ en cinquième semaine).

La situation est relativement similaire en Espagne. Independence Day resta quatre semaines en tête du box office. Après 10 jours d'exploitation, Independence Day battait des records de fréquentation. Ses recettes commencent à chuter à partir de la troisième semaine. Le film figure à la troisième place après cinq semaines, à la septième place après six semaines, à la neuvième place après sept semaines et disparât ensuite du Top Ten. 
En Belgique, Independence Day réussit une excellente première semaine et s'accaparait $50 \%$ du marché. Mais dès sa deuxième semaine, il perdit $35 \%$ de ses entrées.

En Allemagne, rnalgré son fulgurant succès et contrairement aux prévisions, Independence Day ne clépassa pas le cap symbolique et jamais atteint de 1000 copies. Après cinq semaines à la première place et trois semaines d'euphorie (une moyenne de près de 12 millions de dollars par semaine), Independence Day s'essouffla et perdit plus de $40 \%$ d'entrées en une semaine.

11 "On peut raisonnablement parier que le film atteindra - voire dépassera 2,5 millions d'entrées en première semaine", notait Le Film français (n“2633, p. 38). Independence Day attira exactement 2110017 spectateurs en France. Au tableau des meilleures premières semaines, Jurassic Park (2 256888 entrées) garde la première place devant Indepenáence Day. Sur Paris-périphérie, c'est Rambo 2 qui conserve la première place (510096 contre 444932 entrées pour Independence Day).

12 De même, Jurassic Park avait réalisé un démarrage explosif, mais ne figure qu'en dix-neuvième place du box office des 20 dernières années. Par contre, E.T., qui n'avait attiré "que» 1207881 spectateurs en France en une semaine, réalisa finalement près de neuf millions d'entrées. On retrouve une situation identique avec des films français: Trois Hommes et un couffin (66000 entrées sur Paris-périphérie après une semaine et total en France de 10250000 entrées); Le Grand Bleu (97000 entrées sur Paris-périphérie après une semaine et total en France de neuf millions d'entrées).

13 Sociologue américain (1922-1982). Selon Goffman, les individus sont des acteurs en représentation qui jouent des rôles sociaux et donnent aux autres une image valorisée d'eux-mêmes.

14 Durant l'été 1996, des Africains sans papiers s'étaient réfugiés dans l'église parisienne Saint-Bernard, certains menant une grève de la faim. Ils exigeaient du gouvernement français leur régularisation et l'obtention de papiers, affirmant notamment qu'ils étaient en France depuis de nombreuses années et que certains d'entre eux étaient parents d'enfants français ou nés en France. Les immigrés étaient soutenus par plusieurs artistes et intellectuels français. Néanmoins, cette affaire, qui fit grand bruit, divisa l'opinion publique, car elle concernait plus largement la politique d'immigration de la France. Finalement, le gouvernement adopta une solution de compromis, conciliant fermeté et humanité: certains Africains sans papiers furent régularisés, d'autres furent renvoyés dans leur pays d'origine.

15 "Si Independence Day aide à la rélection de Clinton, tant mieux. [...] Bill Clinton nous a servi de modèle. Pourquoi Clinton? En réaction aux présidents escrocs, débiles, croulants qui envahissent les écrans", soulignent Roland Emmerich, le réalisateur, et Dean Devlin, le producteur, dans une interview accordée à L'Express (n" 2360, 26 septembre 1996, p. 113).

16 Allusion à la guerre du Golfe, très présente également dans les critiques comme symbole de l'union du monde autour des intérêts états-uniens. "Exterminer une armée d'extraterrestres est une autre paire de manches que de massacrer des milliers d'Irakiens à l'aveuglette " écrit par exemple Télérama (n" 2438, 2 octobre 1996, p. 32).

\section{CORPUS CRITIQUE}

"Box-office hebdo international". Le Film français, n" 2631 (20 septembre 1996), p. 30.

"Box-office hebdo international". Le Film français, n' 2632 (27 septembre 1996), p. 30 .

"Les films en tête cette semaine". Le Film français, n" 2633 (4 octobre 1996), p. 38.

La réception du film Independence Day en France. Un exemple de contre-acculturation? 
"Box-office hebdo international ". Le Film français, n" 2633 (4 octobre 1996), p. 40. "I ess films français font de la résistance". Le Film français, n" 2634 (11 octobre 1996), p. 28.

"ID4: la semaine de la riposte". Le Film français, n" 2634 (11 octobre 1996), p. 28.

"Box-office hebdo international". Le Film français, n" 2634 (11 octobre 1996), p. 30.

"L.es films en tête cette scmaine". Le Film français, n" 2635 (18 octobre 1996), p. 22.

"Box-office hebdo international". Le Film français, n" 2635 (18 octobre 1996), p. 24.

"L.es films en tête cette semaine". Le Film français, n" 2636 (25 octobre 1996), p. 36.

"Box-office hebdo international ". Le Film français, n" 2636 (25 octobre 1996), p. 38.

"L.es films en tête cette semaine». Le Film français, n" 2637 (1 novembre 1996), p. 20.

"Quatre blockbusters dans le vent". Le Film français, n" 2637 (1 novembre 1996), p. 20.

"Box-office hebdo international". Le Film français, n" 2637 (1 novembre 1996), p. 22.

"Les films en tête certe semaine". Le Film français, n" 2638 (8 novembre 1996), p. 24 .

"Leur part de marché hebdomadaire". Le Film français, n" 2638 (8 novembre 1996), p. 24.

"Box-office hebdo international". Le Film français, n" 2638 (8 novembre 1996), p. 26.

"Box-office cumulé USA 1996". Le Film français, n" 2638 (8 novembre 1996), p. 26. "Spécial Independence Day, Canal Jimmy". TV Hebdo, supplément du Provençal et du Méridional (28 septembre 1996).

Baignères, Claude. "Les extraterrestres font recette». Le Figaro, n" 16220 (10 octobre 1996), p. 30.

Bauberger, Jeannc, Frank Esposito et Jean-Michel Gardanne. "Independence Day. Qu'en pensent-ils?". Sortir, supplément du Provençal et du Méridional $(9$ octobre 1996), p. 12.

Charnay, Dominique. "Independence Day". Télé Star (30 septembre 1996), p. 120.

Dacbert, Sophie. "ID4: la déferlante?". Le Film français, n" 2631 (20 septembre 1996), p. 3.

Esposito, Frank. "Le jour le plus long ". Sortir, supplément du Provençal et du Méridional (2 octobre 1996), p. 10.

Fsposito, Frank. "Independence Day". Sortir, supplément du Provençal et du Méridionull (4 octobre 1996), p. 10.

Forestier, François."Les nouveaux E.T.: bêtes et méchants". Le Nouvel Observateur, n" 1664 (26 septembre 1996), p. 114-115.

Frodon, Jean-Michel. "Independence Day». Le Monde, n" 16064 (19 septembre 1996), p. 28.

Grousset, Jean-Paul. "Independence Day". Le Canard enchaîné, n" 3962 (2 octobre 1996), p. 6.

Krohn, Bill. "'Tornades, Martiens et ordinateurs». Cabiers du cinéma, n" 505 (septembre 1996), p. 55-65.

l atil, Georges. "Independence Day". Le Méridional (4 octobre 1996), p. 28.

l. cfort, Gérard. "Independence niais». Libération, n" 4780 (2 octobre 1996), p. 29.

Martino, Claude. "Alien-Nation!". La Marseillaise (2 octobre 1996), p. 1 et 20. 
Médioni, Gilles. "Ça cartonne à Washington". L'Express, n" 2360 (26 septembre 1996), p. 112-113.

Merrick, Hélène. "Independence Day". Télé Laisirs, n" 533 (30 septembre 1996).

Meyer, Philippe. "Box-office». Le Point, n" 1254 (28 septembre 1996), p. 132.

Mulard, Claudine. "Independence Day, le jour où la Terre fut sauvée par un citoyen américain". Le Monde (3 octobre 1996), p. 27.

Portes, Florence. «Independence Day». Paris Match (19 septembre 1996), p. 16.

Rauger, Jean-François. "Débauche d'images et de stéréotypes". Le Monde (3 octobre 1996), p. 27.

Remy, Vincent. "Independence Day". Télérama, n" 2438 (2 octobre 1996), p. 32.

Tobin, Yann. "Independence Day». Positif, n" 428 (octobre 1996), p. 45.

\section{OUVRAGES CITÉS}

Amselle, Jean-Loup. Logiques métisses. Anthropologie de lidentité en Afrique et ailleurs. Paris: Payot, 1990.

Boltanski, L. Les Cadres. Paris: Les Éditions de Minuit, 1982.

Goffman, Erving. "Lordre de l'interaction", Les Moments et lettrs hommes. Paris: Seuil/Minuit (1988), p. 186-230.

Jauss, Hans Robert. Pour une esthétique de la réception. Paris: (allimard, 1978. 